LA EXTENSIÓN UNIVERSITARIA COMO GENERADORA DE LA FORMACIÓN EXPERIENCIAL. UN RETO EN LA FORMACIÓN UNIVERSITARIA. PÁginas 117-128 en ReVista de la Escuela de Ciencias de la Educación, año 10, número 9, enero a diciembre de 2014. ISSN 1851-6297. ISSN EN LINEA 2362-3349.

\title{
LA EXTENSIÓN UNIVERSITARIA COMO GENERADORA DE LA FORMACIÓN EXPERIENCIAL. UN RETO EN LA FORMACIÓN UNIVERSITARIA
}

\author{
Por Roberto Luis Albarenque (Universidad Nacional de Entre Ríos)*, Argentina. \\ roalbaren@yahoo.com.ar \\ María Virginia Walz \\ (Universidad Nacional de Entre Ríos - Universidad Autónoma de Entre Ríos)**, \\ Argentina. \\ virginiawalz@bioingenieria.edu.ar
} Recibido: 02/07/2014 Aceptado: 28/07/2014

\section{Resumen}

Pensar en la incorporación en el proceso formativo de los estudiantes universitarios de intervenciones e interacciones sociales relacionadas a su práctica profesional es de suma importancia. Privilegiar el "aprender haciendo" y el aprendizaje a través de la experiencia, como sostiene J. Dewey (1916), debería ser una iniciativa del trabajo académico docente desde un principio, desde cada una de las etapas del proceso educativo, desde la planificación, cronogramas y desarrollos, por un lado, y por otro lado, del proceso de aprendizaje del estudiante, de su crecimiento educativo, de su mejoramiento en la comprensión de los contenidos académicos, del desarrollo de habilidades y capacidades de iniciativas para resolver efectivamente necesidades que en su futuro le demande la comunidad. La tensión que genera hoy en día jerarquizar la extensión en aquel contexto formativo, es una discusión que está vigente en el ámbito universitario.

\section{Palabras Clave}

Extensión - Curricularización - Formación experiencial - Formación integral.

* Analista Programador de Sistemas. Docente de la Licenciatura en Educación y del Profesorado en Educación de la Facultad de Ciencias de la Educación de la Universidad Nacional de Entre Ríos.

** Master en Ingeniería Biomédica. Bioingeniera (Facultad de Ingeniería de la Universidad Nacional de Entre Ríos). Docente de grado de la Facultad de Ingeniería de la Universidad Nacional de Entre Ríos y de la Facultad de Ciencia y Tecnología de la Universidad Autónoma de Entre Ríos. 
Revista de la Escuela de Ciencias de la Educación, año 10, número 9, enero a diciembre de 2014. Páginas 117-128. ISSN 1851-6297. ISSN EN LINEA 2362-3349. LA EXTENSIÓN UNIVERSITARIA COMO GENERADORA DE LA FORMACIÓN EXPERIENCIAL. Un reto en la Formación universitaria. Roberto Luis Albarenque - Maria Virginia Walz.

\begin{abstract}
Think about the incorporation of social interventions and social interactions related to the professional practice in the training process of university students is very important. To privilege the "learning by doing" and learning through experience, as Dewey (1916) holds, should be a initiative of the academic work from the beginning, from each of the stages of the education process, from academic planning, schedules and developments, on the one hand, and, on the other hand, of the learning process of the student, of their educational growth, of the better understanding of the student of the academic content, of the development of skills and capabilities for initiative to effectively resolve in the future necessities that the community demands it. The tension that today generates hierarchize the extension in that learning context, is a current discussion at the university environment.
\end{abstract}

\title{
Key Words
}

Extension - Curricularization - Experiential training - Integrated training.

\section{Introducción}

\section{La integración de la educación experiencial en el currículo universitario}

La extensión universitaria, según lo define el Consejo Interuniversitario Nacional (CIN) (1), implica una interacción con sectores sociales que surge de diferentes supuestos y enfoques políticos e históricos, poniendo en juego saberes y valores en la interrelación universidad-sociedad. Este carácter sustantivo de la extensión se refleja fundamentalmente en los estatutos de la mayoría de las universidades nacionales, donde se la caracteriza como uno de los pilares o funciones principales junto a la docencia y la investigación, ubicando a estas tres dimensiones en un mismo plano de igualdad.

En vinculación a esto, la Universidad Nacional de Entre Ríos (UNER) (2), una de las instituciones educativas en las que nos desarrollamos profesionalmente, sostiene que la extensión constituye un canal de comunicación permanente con la sociedad a partir de su desarrollo académico, científico y tecnológico, comprometiéndola con una misión de tarea democrática y de transformación política, social y económica, para lo cual debe existir un diálogo democrático, interactivo y multidireccional con la sociedad, promoviendo el desarrollo integral de la misma. Por su parte, otro ejemplo que podemos citar es la visión de la Universidad Autónoma de Entre Ríos (UADER) (3) que tiende al desarrollo regional sustentable mediante capacitación en servicio, investigación tecnológica y de desarrollo que atienda a la demanda social y productiva de la región, brindar servicios tecnológicos y de transferencia articulando su accionar con el sector empresario.

En particular, la Facultad de Ciencias de la Educación (FCEDU) (4) de la UNER adhiere a la idea de la extensión de la siguiente manera: 
Revista de la Escuela de Ciencias de la Educación, año 10, número 9, enero a diciembre de 2014. Páginas 117-128. ISSN 1851-6297. ISSN EN LINEA 2362-3349. LA EXTENSIÓN UNIVERSITARIA COMO GENERADORA DE LA FORMACIÓN EXPERIENCIAL. Un reto en la formación universitaria. Roberto Luis Albarendue - María Virginia Walz.

(...) como democratización del saber, como función social de una universidad abierta, critica y creativa. En este sentido, mediante la extensión se propone establecer un diálogo interactivo y multidireccional que aporte al crecimiento cultural y a la transformación social (...) La extensión dinamiza el vínculo entre universidad y sociedad por ser un elemento integrador que facilita el intercambio de ideas, la construcción de saberes y promueve el desarrollo integral partiendo del trabajo con el otro, de forma articulada (...) De igual manera que los procesos curriculares o de investigación, los de extensión universitaria constituyen la arena en que se producen intercambios socio-culturales y en simultáneo se gestan identidades, en la que se producen marcos de referencia y actuación que inciden en las relaciones de los sujetos para con otros y con sí mismos (4).

Desde el entendimiento de la Facultad de Ingeniería (FI) (5) de la UNER, ésta concentra sus esfuerzos en acciones que dan cuenta de su presencia e interacción académica en la sociedad en general, aportándole los resultados de su investigación y docencia en el marco de sus saberes y habilidades específicas realizando actividades que le permitan identificar problemas y demandas de la sociedad.

La perspectiva de la Facultad de Ciencia y Tecnología (FCYT) (2) de la UADER respecto a la extensión universitaria es buscar desde un proceso dinámico el aporte de respuestas a situaciones concretas de la realidad, enriqueciendo las prácticas de docentes y estudiantes.

A partir de la concepción de la UNER y UADER sobre la extensión universitaria y su posicionamiento respecto al CIN, nos preguntamos: ¿la equidad de prioridades entre las funciones sustantivas se manifiestan en las políticas de la institución de una manera sistemática?, ¿las políticas se ven reflejadas en acciones concretas de extensión hacia un colectivo social? La dinámica del vínculo entre la sociedad y la universidad, ¿es interpretada desde ambas partes de igual manera? ¿La institución tiene una idea clara de lo que se entiende por formación experiencial integral?

Asimismo, entre muchísimas otras miradas desde una perspectiva de posible acción protagónica de la universidad hacia un colectivo social, al analizar otros aspectos desde el interior de la institución educativa ¿qué apreciación de la inserción académica de esta función (curricularización de la extensión universitaria) se hace desde cada disciplina?, ¿está presente en las cátedras?, ¿se incorpora la articulación entre extensión, docencia e investigación en la propuesta de cátedra?, ¿el docente tiene una visión formada del trabajo cooperativo con la sociedad desde su cátedra y viceversa con participación activa del alumno?

Desde otro plano de interrogantes, es curioso que cuando se tiene que declarar el desempeño docente, éste quede definido bajo la categoría docen- 
Revista de la Escuela de Ciencias de la Educación, año 10, número 9, enero a diciembre de 2014. Páginas 117-128. ISSN 1851-6297. ISSN EN LINEA 2362-3349. LA EXTENSIÓN UNIVERSITARIA COMO GENERADORA DE LA FORMACIÓN EXPERIENCIAL. Un reto en la Formación universitaria. Roberto Luis Albarenque - Maria Virginia Walz.

te - investigador. Ejemplos de ello: planillas de incentivos, categorizaciones, llamados a becas, intercambios, etc. Entonces, ¿cómo relacionar esta denominación generalizada con la extensión?, ¿se puede considerar al docente como docente - investigador - extensionista en el camino hacia una formación experiencial del alumno?

Es ingenuo pensar que todos estos cuestionamientos, como muchos otros, no surjan continuamente en las unidades académicas cuando se trata el tema de la extensión y más específicamente de algo tan poco practicado por tradición universitaria como lo es un proceso de educación basada en la experiencia. Infinidad de cuestiones y respuestas se debaten y analizan, pero en su mayoría no son afines a una visión de formación en extensión y mucho menos a la búsqueda de acciones para concretizarla. Frente a esto, el docente, tarde o temprano, tendrá que autoreflexionar para lograr internalizar una nueva concepción de su actividad docente, teniendo en claro por un lado y entre otras tantas cuestiones, que a la hora de planificar su actividad académica el incluir prácticas extensionistas corresponderá a aceptar el completo involucramiento del alumno en esa planificación desde un principio; y, por otro lado, conocer sin dudas que al alumno lo expondrá frente a una situación nueva, concreta, real, ajena y distinta a su acostumbrada cotidianidad universitaria, que le demandará comprometerlo en su totalidad, requerir de su compromiso personal y responsabilidad cívica para asumir roles significativos y desafiantes frente a la necesidad de responder eficazmente a lo que la situación le demande.

Este trabajo tiene como objetivo analizar el propio discurso desde la inclusión de la educación experiencial como el componente distinto dentro del programa de formación.

\section{Breve reseña de la incorporación de la extensión en la universidad}

En verdad, son cuestiones que datan desde principio del 1800 con los primeros momentos de la incorporación de la extensión a la universidad, donde los actores de diversos sectores sociales comenzaron a "golpear sus puertas", las que casi siempre permanecían cerradas al medio, requiriendo acciones concretas tendientes a difundir el conocimiento generado por el aporte económico de toda la sociedad en su conjunto. Desde un ángulo acotado solamente al ámbito académico universitario, el movimiento de la clase media protagonizó con el deseo de hacer valer su derecho de poder acceder a una formación superior, reservada sólo a la clase pudiente de la época con su estructura de academia señorial. Situaciones de confrontación entre la sociedad y los cerrados esquemas universitarios del comienzo del siglo XX desencadenaron trascendentes sucesos que fueron dando forma al actual concepto de extensión. En Latinoamérica y en particular en Argentina, y sin lugar a dudas, la Reforma Universitaria de 1918 surgida en la ciudad de Córdoba, da cuerpo a la función de extensión, en toda su dimensión y con características propias, formando 
Revista de la Escuela de Ciencias de la Educación, año 10, número 9, enero a diciembre de 2014. Páginas 117-128. ISSN 1851-6297. ISSN EN LINEA 2362-3349. LA EXTENSIÓN UNIVERSITARIA COMO GENERADORA DE LA FORMACIÓN EXPERIENCIAL. Un Reto en la formación universitaria. Roberto Luis Albarenque - María Virginia Walz.

parte de la labor permanente de la institución universitaria, entendida como la tercera misión que se integra junto a la enseñanza e investigación al conjunto de actividades universitarias. A partir de 1972 en la Segunda Conferencia Latinoamericana de Extensión Universitaria y Difusión Cultural, surge un nuevo concepto de extensión universitaria modificando el rol de la universidad con la inclusión de la función social en su clásica misión, llevándola a entablar y conciliar, con el paso del tiempo y hasta el día de hoy, un sólido vínculo con la sociedad y su problemática, madurando en la creación de espacios de articulación entre ambas identidades. De esta manera, este inédito perfil es parte de la historia de la definición de una universidad latinoamericana nueva que marca un vector a sus actores sobre la idea del ejercicio de una proyección social de su labor, mediante una tarea democrática y abierta, sin restricciones ideológicas, traducida en momentos de mayor concientización y permeabilidad del quehacer universitario en relación a su entorno social con el objeto de lograr un mejor nivel académico. En este mismo sentido, desde la UNESCO (6) se sostiene que la educación superior debe avanzar en una configuración activa de relación con sus contextos y trabajando en conjunto con las comunidades, indagando sus problemáticas para poder atenderlas con soluciones concretas derivadas de tareas de investigaciones científicas, tecnológicas y artísticas, encaradas por la motivación de conocer explícitamente el problema a atender.

Dora Barrancos (1993) plantea la extensión como una especie de "contaminación ambiental" que la universidad genera en la sociedad al querer acercar la cultura y la ciencia al colectivo social. Risieri Frondizi (1987) opina que la universidad no puede ser ajena a la realidad, a los problemas ni a las demandas de la sociedad en que vive y que la función social de la universidad se ve reflejada en su acción extensionista al extender a las masas los conocimientos que se imparten en aquella. En concordancia, Sousa Santos (2006) enmarca la necesidad de un compromiso político de la universidad con la sociedad que la involucra.

\section{Una visión acerca del currículo en lo que llamaríamos educación experiencial}

Pensar en la incorporación en el proceso formativo de los estudiantes de carrera de grado de intervenciones e interacciones sociales relacionadas a su práctica profesional es de suma importancia. La tensión que genera hoy en día jerarquizarlas en contexto requiere desplegar nuevos espacios interdisciplinarios de aprendizaje y servicio en los que necesariamente se ampliarán conocimientos o se generarán nuevos y se desarrollarán nuevas habilidades y capacidades en sintonía con el medio con el que se opera. En torno a esto, existen variadas propuestas de formación en extensión para brindarle al alumno un marco teórico y metodológico que lo ayude a articular estudio y trabajo tendientes a lograr una formación profesional integral, profundizando el compromiso con la 
Revista de la Escuela de Ciencias de la Educación, año 10, número 9, enero a diciembre de 2014. Páginas 117-128. ISSN 1851-6297. ISSN EN LINEA 2362-3349. LA EXTENSIÓN UNIVERSITARIA COMO GENERADORA DE LA FORMACIÓN EXPERIENCIAL. Un reto en la Formación universitaria. Roberto Luis Albarenque - Maria Virginia Walz.

sociedad. Por ejemplo, desde 2007, la Universidad de la República, a partir de su Segunda Reforma Universitaria, ha incorporado la extensión al conjunto de la oferta educativa materializado por prácticas integrales separadas en espacios de formación experiencial, y la Universidad Nacional del Litoral, también desde 2007, incluye prácticas en extensión en carreras de grado. En la UNER todavía no hay una propuesta consensuada de incluir en toda la oferta educativa las actividades de extensión y una formación experiencial explícita, pero sí cabe mencionar que existe una fuerte tendencia que ya se refleja en la definición de nuevas políticas de medidas concretas y de impacto en plena concordancia con lo pactado en el estatuto del CIN en la conformación de un plan estratégico de extensión universitaria. En este sentido, por ejemplo, la UNER es una de las pioneras en innovar institucionalmente al establecer la evaluación externa de pares, con instancia de entrevista presencial incluida. Asimismo, el incremento en el presupuesto para la ejecución de acciones, proyectos y programas de extensión, favoreciendo la ampliación de las posibilidades de cooperación entre la universidad y el medio social, es altamente notable de la nueva posición y consolidación que va tomando gradualmente la extensión.

Una visión jerarquizadora de la actividad extensionista es pensar en que estas intervenciones podrían transformarse en estudios de campo que sirvan como materia prima para la investigación. En consecuencia, la extensión puede ser una herramienta no sólo de vinculación, sino de interpelación a la práctica y política universitaria, así como de indagación a los supuestos que subyacen a la apropiación social de los conocimientos y la construcción social de los mismos. Para conformar un plan de acción para la curricularización de la extensión son necesarios consensos básicos comunes a toda la comunidad universitaria, que la atraviesen vertical y horizontalmente, para su consecución (7).

Un buen diálogo de la universidad con la sociedad hacia un buen encuadre de trabajo mutuo vale más que mil recetas y supuestos de integración entre ésta y el colectivo social, involucrando a los actores en actividades que los estudiantes en cooperación realizan al servicio de la comunidad, aplicando sus habilidades y conocimientos académicos y profesionales para dar respuesta, eficaz y valorada, a los requerimientos específicos de aquella. Esta filosofía educativa, que internacionalmente recibe el nombre de "aprendizaje-servicio" o "aprendizaje en servicio" (en inglés: service-learning), atiende por igual estrategias que apunten a garantizar un servicio de calidad y estrategias de enseñanza que focalicen específicamente a un aprendizaje de calidad. Privilegiando el "aprender haciendo" y el aprendizaje a través de la experiencia, como sostiene J. Dewey (1916), en esta iniciativa de trabajo la participación de todos, docentes y alumnos, es desde el principio, desde las etapas de diagnóstico, planificación, cronograma y desarrollo (acorde al calendario académico) hasta las de gestión y evaluación tanto de la ejecución y de los resultados del proyecto como del proceso de aprendizaje del estudiante, de su crecimiento educativo, 
Revista de la Escuela de Ciencias de la Educación, año 10, número 9, enero a diciembre de 2014. Páginas 117-128. ISSN 1851-6297. ISSN EN LINEA 2362-3349. LA EXTENSIÓN UNIVERSITARIA COMO GENERADORA DE LA FORMACIÓN EXPERIENCIAL. Un reto en la formación universitaria. Roberto Luis Albarendue - María Virginia Walz.

de su mejoramiento en la comprensión de los contenidos académicos, del desarrollo de habilidades y capacidades de iniciativas para resolver efectivamente la demanda sentida por la comunidad, preservando en todos los casos el rigor académico-profesional por los aspectos que el alumno aprende y lo capacitan para utilizarlos, y que le agrega valor a su formación como ciudadano partícipe y en el desarrollo de actitudes solidarias.

John Dewey (1938) describe un marco de referencia permanente entre educación y experiencia personal como un binomio que tiene, por un lado, armonía y consonancia, y por el otro, cada una de ellas concibe lo enseñado de maneras diferentes. A su vez menciona que no alcanza con insistir en actividades basadas en la experiencia sino que todo depende de la calidad con que dichas experiencia se han realizado. John Dewey habla de experiencia educativa en la que la educación es aquella reorganización o reconstrucción de la experiencia que da sentido a la experiencia y que aumenta la capacidad para dirigir el curso de la experiencia subsiguiente. Además, agrega que las experiencias no son ni buenas ni malas, depende del sujeto y de la circunstancia que sean una cosa o la otra. A partir de esta concepción, surge que en la interacción con el mundo real, innovamos ideas que nos permiten vivir mejor, sobrevivir, adaptarnos mejor, sensibilizando nuestro juicio que es la expresión de la experiencia y que además si este juicio permite seguir adelante con los propios valores, entonces el juicio es verdadero aunque esto no significa que la realidad sea así sino sólo una perspectiva de la misma.

La presentación de una situación concreta, compleja, no simplificada, compromete al alumno en su totalidad alejándolo de la pasividad, consiguiendo que explore y descubra por sí mismo sus propias actitudes y requiere de su compromiso personal y responsabilidad cívica para asumir roles significativos y desafiantes frente a la necesidad de responder eficazmente a una demanda de la comunidad, que previamente se conoce, pero de la que también pueden acontecer situaciones no previsibles y/o ambientes desconocidos con posibilidad de riesgos no anticipados, demandando aprendizaje de nuevos conocimientos y del saber aplicarlos, integrado a lo que se conoce.

Una formación experiencial apuntaría a ofrecerle al alumno un aprendizaje íntegro y auténtico y una experiencia significativa de servicio, que lo ayude a aprender más y mejorar su aprendizaje académico, facilitando el fortalecimiento de los procesos de diálogo y acuerdo al verse expuesto frente a una situación nueva.

Algunos objetivos que podrían considerarse para la formación experiencial mediada por la actividad extensionista del estudiante serían, por ejemplo, fortalecer prácticas de interacción e intervención en el colectivo social, lograr el mejoramiento de la formación académica y profesional de los estudiantes promoviendo la articulación estudio-trabajo-sociedad, ampliar los saberes disciplinares articulando con actividades inter- y transdisciplinarias, retroali- 
Revista de la Escuela de Ciencias de la Educación, año 10, número 9, enero a diciembre de 2014. Páginas 117-128. ISSN 1851-6297. ISSN EN LINEA 2362-3349. LA EXTENSIÓN UNIVERSITARIA COMO GENERADORA DE LA FORMACIÓN EXPERIENCIAL. Un reto en la Formación universitaria. Roberto Luis Albarenque - Maria Virginia Walz.

mentar con los conocimientos adquiridos en la práctica experiencial los planes curriculares de los espacios de conocimiento involucrados por un lado, y en la actividad profesional, por el otro. Asimismo, lograr formar un espíritu crítico cuidadoso, deliberado, centrado en un objetivo orientado a la obtención de un resultado, incluir en los planes de cátedra cronogramas de actividades en donde se contemple el tiempo necesario para que los actores involucrados reflexionen sobre la experiencia de servicio para una posterior puesta en común que dinamice la intercomunicación y el trabajo en equipo, visibilizar mecanismos claves para un diálogo fluido y sostenido de comunicación entre los distintos participantes del presente y futuros, formar hábitos solidarios y de buen uso del conocimiento y de calidad, desarrollar una mentalidad abierta a situaciones no previsibles, entre otras.

Frente a todo lo expuesto, es evidente que la formación experiencial curricular necesita modos de trabajar diferentes tanto del alumno como del docente. Estos nuevos modos exigen trabajar en nuevos diseños de programas de formación y de su implementación, o bien en adaptar los ya existentes adoptando a la educación experiencial como componente distinto y relevante de lo que ya está escrito como diseño curricular. Para que la educación experiencial sea una propuesta pedagógica, primeramente debe sobrevenir un proceso de cambio en la representación mental que el docente posee, para ir logrando un convencimiento absoluto de que este tipo de enseñanza y aprendizaje es una mirada totalmente diferente de la educación tradicional, que es una nueva concepción filosófica que debe aceptar e interiorizar para poder ponerla en práctica. En esto, la función del docente se traduce en lograr que el alumno aprenda haciendo, aprenda de sus errores, reflexione sobre el hacer modificando retroactivamente sus criterios y estrategias para readecuar sus acciones en una circunstancia determinada y en futuras. Una buena práctica se vale del conocimiento, de las habilidades, de los valores y de la ética y es el docente el que debe buscar la manera de incluir estos cuatro componentes en la formación integral de sus alumnos, teniendo en claro que el valor social y el beneficio para la sociedad nutren al proceso de trabajo social auténtico.

Una asignatura es un conjunto de contenidos a enseñar que surge de la construcción de un currículo a partir de diferentes recortes del universo de conocimiento de una disciplina o de varias, cada una con justificado valor pedagógico, que al incluirle un nuevo elemento de formación su andamiaje tendría que estructurarse de manera tal de contener a la formación experiencial como algo natural y manteniendo el perfil del graduado. Hablamos de una formación experiencial integral siempre y cuando no haya una fragmentación de las asignaturas de la carrera universitaria y para esto en el diseño curricular que la cátedra construye, además de marcar las pautas propias de la disciplina a la que pertenece, sería apropiado buscar tanto canales de comunicación que favorezcan el intercambio de experiencias constructivas de sus partes dando 
Revista de la Escuela de Ciencias de la Educación, año 10, número 9, enero a diciembre de 2014. Páginas 117-128. ISSN 1851-6297. ISSN EN LINEA 2362-3349. LA EXTENSIÓN UNIVERSITARIA COMO GENERADORA DE LA FORMACIÓN EXPERIENCIAL. Un reto en la formación universitaria. Roberto Luis Albarendue - María Virginia Walz.

forma al concepto de unidad y sin perder su propia lógica, así como enseñar las condiciones sociales en las cuales el conocimiento y el saber se van a utilizar, intentando disminuir el déficit en este sentido que la formación superior, en la mayoría de las veces, suele tener.

Asimismo, el plantearse una formación distinta basada en la experiencia y en la extensión de saberes necesita de la reflexión sobre el fortalecimiento de la educación universitaria en lo que respecta a la utilidad de las cosas que se enseñan. Bajo esta línea, algunos de los contenidos curriculares tienen que ser enriquecidos con un significado social poderoso para poder ser usado y transferido a la solución de problemas concretos de grupos sociales diferentes con el objeto de mejorar la calidad de vida de ellos. Es un compromiso a tomar por las instituciones educativas de cambiar el prejuicio cognitivo acerca de la importancia social de los alumnos en el contexto universitario pues la universidad está sostenida por lo social y debe ser proactiva con ella. Estos cambios podrían fortalecer el campo de acción del alumno, ampliando su perspectiva conducente a una apertura mental que le permita resolver cuestiones desde diferentes puntos de vista y quizás le permita desarrollar grandes capacidades para encarar tanto nuevos problemas como diversos que a larga se traduzcan en impactos reales a la sociedad. Capacidades mediadas por aprendizajes genuinos derivados de la propia experiencia con la interacción social conjugada con los valores éticos y morales de las personas y no de una mera práctica de pizarrón, absolutamente simplificada e idealizada de la vida real.

La práctica áulica cotidiana, es cerrada, previsible, arreglada, elegida y por ende no le permite al alumno aprender de la imprevisibilidad de una situación real, dando lugar a una percepción falsa y equivocada de lo que es el desempeño profesional auténtico y provocando de manera involuntaria grados de responsabilidad no iguales a los que se presentarían al trabajar con casos reales. La experiencia se va formando con lo que se vive, se hace, cómo se hace y para qué se hace, imprimiendo una dinámica en la que los conocimientos no son estáticos sino que evolucionan, transmutan o se transforman (lo que se sabe hoy acerca de algo no tiene por qué ser lo mismo a lo que se sabrá mañana), entre otras cuestiones que en general no pertenecen a una clase carente de mediación experiencial. Esto permitiría, tal vez, cambiar el concepto de transferencia larga de conocimiento en la que el alumno se nutre de conceptos y enunciados teóricos y que es habitualmente entendida como una herramienta de trabajo y destreza de alcances no percibidos en una instancia temprana de aplicación; dicho más simple, el alumno al no encontrar justificación pedagógica y pronta aplicación, no puede entender por qué y para qué se le enseñan ciertos temas. En este contexto se debería hablar de transferencia de sentido inmediato construido por la propia experiencia en lugar de transferencia larga, teniendo presente que no es un proceso automático ni repetitivo sino que obliga a formular conceptos y requiere de una evolución reflexiva, específica 
Revista de la Escuela de Ciencias de la Educación, año 10, número 9, enero a diciembre de 2014. Páginas 117-128. ISSN 1851-6297. ISSN EN LINEA 2362-3349. LA EXTENSIÓN UNIVERSITARIA COMO GENERADORA DE LA FORMACIÓN EXPERIENCIAL. Un reto en la Formación universitaria. Roberto Luis Albarenque - Maria Virginia Walz.

e interpretada, no general, tanto del alumno como del docente para validar el aprendizaje y su significado desde sus propias perspectivas contextualizadas. Es un proceso de transacción entre ambos actores por el cual se benefician mutuamente "le enseño-me enseña" llevando sus bitácoras a un estado superior de conocimiento, a partir de usar la habilidad de pensamiento crítico y creativo, que no es sinónimo de inventivo, que les concede la capacidad de mirar la situación en contexto y entonces actuar diferente ante esa condición problemática, que no es rutinaria, para brindar un servicio de solución.

Al tratarse de una enseñanza diferente, de tinte holístico, está orientada a vincular el aprendizaje académico con los sucesos de la vida real, que no son neutros, conectando ambas dimensiones con actividades en la que los estudiantes intencionalmente implicados en experiencias directas se ponen a prueba, actúan en consecuencia y se evalúan per-se por los resultados de sus propias acciones e iniciativas previamente tomadas, que podrán ser exitosas o desafortunadas. El conocimiento queda marcado por la conceptualización de la situación en la que fue aplicado y por el significado social que ésta tiene, obviamente desde la perspectiva del alumno pero acompañada con la del docente. Por esto, una planificación que contemple alguna forma de contacto con un ambiente de práctica social real, de la creación conjunta de un dispositivo de acción entre el docente, el alumno y el conocimiento en común en respuesta a la demanda surgida, puede ser uno de los motivos por el que el estudiante se realice en contextos similares a los que se enfrentará al egresar y de esta manera, en este sentido, verse favorecido con una formación profesional más integral, más crítica y más comprometida por incluir vivencias de interacciones e intervenciones sociales. Desde este punto de vista, la creación de un espacio que articule estudio y trabajo requerirá del diseño de situaciones de aprendizaje en la extensión, lo que interpelará a las prácticas de la enseñanza y a la búsqueda de innovadoras estrategias pedagógicas, entre muchas otras intervenciones y todas muy distintas a lo que se considera tradicional en muchas carreras y cátedras, y por ende poco atractivo; pero, inevitablemente pondría en diálogo a las funciones de docencia y extensión. Recuperando el concepto de dinamismo planteado anteriormente, las planificaciones que convencionalmente conocemos, si toman este tipo de enseñanza se verán afectadas a un ritmo marcado por los resultados de la constante interacción con la sociedad y estos cambios tendrán que repensarse para adecuarse mejor a las necesidades del grupo social afectado para solucionarlas.

Queda claro que, la educación superior debería perseguir una "práctica integral" bien diseñada donde se articulen además de las tres funciones sustantivas de la universidad: investigación, extensión y docencia, sea prioridad también la definición de nuevas formas de enseñar y construir nuevos conocimientos, tanto hacia el interior del sistema universitario, como con la comunidad, para permitir obtener un enfoque regional y atender las demandas y los problemas locales, 
Revista de la Escuela de Ciencias de la Educación, año 10, número 9, enero a diciembre de 2014. Páginas 117-128. ISSN 1851-6297. ISSN EN LINEA 2362-3349. LA EXTENSIÓN UNIVERSITARIA COMO GENERADORA DE LA FORMACIÓN EXPERIENCIAL. Un reto en la formación universitaria. Roberto Luis Albarendue - María Virginia Walz.

dentro de los principios de sustentabilidad del ambiente, equidad e integración de todos los miembros. Una práctica integral daría origen a una articulación posibilitadora y responsable para la construcción de otros conocimientos que se relacionen con la sociedad y formarían un ambiente que permita promover iniciativas vinculadas con el desarrollo de una sociedad más justa y solidaria junto con el ejercicio de una ciudadanía plena.

\section{A modo de reflexión}

El proceso de revalorización de la extensión está iniciado. La creación de la Red Nacional de Extensión Universitaria (REXUNI) por el CIN en el año 2008 da cuenta de ello al instalarse fuertemente en la agenda universitaria con requerimientos de acción para modelar las diferentes tipologías de extensión que hoy la caracterizan. Con sólo observar las definiciones formales de extensión de las diferentes unidades académicas citadas anteriormente, se pueden notar las numerosas líneas de trabajo que han ido dibujando en la extensión universitaria una gran diversidad y complejidad de actividades y acciones. Esta multiplicidad de enfoques es delineada por los procesos socio-culturales, históricos y políticos, como así también religiosos, que atraviesan desde siempre a la universidad.

Sin embargo, aún son numerosas las posturas más tradicionales (transferencia de conocimientos, proyección de las universidades hacia la comunidad, divulgación cultural y científica, servicios a terceros, transferencia tecnológica, etc.) y otras más actuales y relacionadas con los criterios adoptados por la REXUNI, a saber: diálogo con la comunidad, mutuo aprendizaje, interpelación de saberes, proceso de comunicación entre universidad y sociedad, formación integral, formación experiencial, trabajo cooperativo, etc..

Pensar la incorporación en el proceso formativo de los estudiantes de carrera de grado de intervenciones e interacciones sociales relacionadas a su práctica profesional es de suma importancia. La tensión que genera hoy en día jerarquizarla en contexto, a partir de pensar que de las tres funciones sustantivas de la universidad dos de ellas están curricularizadas, y siendo que conforman un trípode indisoluble pensado no como una sumatoria sino más bien como una integración de funciones para la que cada una de ellas sufrirá necesariamente una transformación, es una discusión que está vigente en el ámbito universitario.

Algunas instituciones educativas de nivel superior ya han incorporado la práctica de extensión al proceso educativo de los estudiantes de carrera de grado. Por otra parte, un encuentro entre la enseñanza y la investigación pondría al alumno en contacto con los generadores de conocimientos diferentes, planteando otras formas de relación con el saber científico y otras formas de participación académica. 
Revista de la Escuela de Ciencias de la Educación, año 10, número 9, enero a diciembre de 2014. Páginas 117-128. ISSN 1851-6297. ISSN EN LINEA 2362-3349. LA EXTENSIÓN UNIVERSITARIA COMO GENERADORA DE LA FORMACIÓN EXPERIENCIAL. Un reto en la Formación universitaria. Roberto Luis Albarenque - Maria Virginia Walz.

Para entender el nexo entre la integración/articulación de dimensión trinómica - docencia, investigación, extensión - es necesario apuntar a la formulación de preguntas y a la obtención de respuestas desde el encuentro entre las tres funciones, para consolidar la demanda e implementar los resultados. Por esto, no se puede pensar la integralidad entre las tres funciones sin incluir los actores sociales, puesto que sin éstos no podemos hablar de demanda y sin aquellos de propuestas que resuelvan problemáticas concretas.

\section{Notas bibliográficas}

(1) Consejo Interuniversitario Nacional. Comisión de Extensión Red Nacional de Extensión Universitaria (REXUNI). Acuerdo Plenario No 681/08.

(2) Proyecto Institucional de la Facultad de Ingeniería de la Universidad Nacional de Entre Ríos (UNER). (2005). Res. CD N²67/04 bis. Manual de Inducción.

(3) Reglamento para Proyectos de Extensión de Universidad Autónoma de Entre Ríos (UADER). (2010). Anexo I de la Ordenanza Nº 028/10.

(4) Secretaría de Extensión de la Facultad de Ciencias de la Educación de la Universidad Nacional de Entre Ríos. Recuperado de http://portal.fcedu.uner.edu.ar/gestion/ secretarias/extension/

(5) Reglamento para la Presentación y Evaluación de Proyectos de Extensión de la Universidad Nacional de Entre Ríos. (2011). Ordenanza Nº 388.

(6) UNESCO. (1997). Las universidades y la educación. El rol de las universidades. Recuperado de http://www.unesco.org/education/uie/confintea/pdf/2a_span.pdf

(7) Secretaría de Extensión de la Universidad Nacional de Entre Ríos. Recuperado de http:// www.extension.uner.edu.ar/sites/default/files/CurriculizacionExtension.doc

\section{Referencias Bibliográficas}

- Barrancos, D. (1993). La 'extensión universitaria': una raíz dormida de la Reforma. Revista Pensamiento Universitario, 1.

- Dewey, J. (1916). Democracia y Educación: Una Introducción a la Filosofía de la Educación. Colección Pedagogía: Morata.

- Dewey, J. (1938). Experiencia y educación. Buenos Aires: Losada.

- Frondizi, R. (1987). Función social de la Universidad. Revista Universidad de la Universidad Nacional del Litoral. Volumen (homenaje).

- Sousa Santos, B. (2006). Renovar la teoría crítica y reinventar la emancipación social. Buenos Aires: UBA/CLACSO.

- Tünnermann C. (2002). El nuevo concepto de la Extensión Universitaria. Cuaderno $N^{\circ}$ 5, Venezuela: Universidad de Carabobo. 